\title{
Modernidad e Identidad
}

Elia Calderón Leyton ${ }^{1}$

Recibido en marzo de 2015, aceptado en abril de 2015.

\begin{abstract}
Resumen
Este ensayo trata sobre las relaciones entre las concepciones de modernidad e identidad en la obra del filósofo Edmund Husserl. En los escritos de Husserl, el concepto de mundo de la vida es una herramienta útil para percibir claramente la concepción del yo, en el contexto de una nueva visión del espacio. En su búsqueda de las fuentes del yo, Husserl centra su análisis en la crisis de la modernidad. Otras consecuencias prácticas de estas distinciones y conceptos de Husserl pueden encontrarse en la intersubjetividad y la cultura.
\end{abstract}

\author{
Palabras claves \\ Modernidad, yo, identidad, crisis, cultura.
}

\begin{abstract}
:
This essay introduces the relationships between the conceptions of identity and modernity in the philosophers Edmund Husserl. In the work of Edumund Husserl, the concept of world is a useful tool in order to see clearly the self in the context of a new vision of the entire domain. In his search of the self, Husserl and center his analysis in the modern epoch. Other practical consequences of the works of Husserl are in the field of intersubjetivity and the culture.
\end{abstract}

Keywords:

Modernity, self, identity, crisis, culture.

Comencemos preguntándonos si la crisis de la modernidad es principalmente una crisis del acceso del sujeto al objeto como lo plantean algunos autores o más bien un problema de acceso al yo. El yo trascendental de Husserl se diferencia de todas las concepciones del yo idealista por tener principalmente el tiempo como condición necesaria para que haya conciencia. Recordemos que la concepción de tiempo cambia según la cultura, del contexto histórico y del tipo de racionalidad (como sucede por ejemplo en la occidental e oriental) en el cual

1. Doctora en filosofía por la Universidad de Humboldt, Berlín. Universidad de Santiago de Chile. 
este se haya inserto. Según Husserl el yo de mi certeza, es un yo del presente, es decir no tiene pasado ni futuro ni le es posible imaginarle un comienzo ni un final. El concepto de conciencia en Husserl encontró una estructura temporal en donde interfiere el pasado y el futuro como proyecciones de ese presente mismo, de su flujo vivido en forma inmediata. Este flujo, está compuesto por el ahora mismo, el aun y el ya enseguida. Es por ello que en esta estructura se pierde la psicología del yo individual y mundano.

"Yo soy yo trascendental de una vida trascendental, de habitualidades trascendentales emanadas de allí, donde en el fluir trascendental se constituye mundo como sentido del ser, y de tal modo que en el a su vez se ha constituido y se sigue constituyendo esta vida trascendental misma como mundanizada: en forma de mi conciencia humana (en sentido lato, de vida del conocimiento)".

El ámbito egologico está determinado por la coherencia de lo que hay en ella. Esta coherencia se supone pero no es suficiente para el concepto de realidad del mundo con independencia de la conciencia. Lo que unifica la experiencia de si y la experiencia del otro, lo que hace que uno sea el a priori del otro es la debilidad de la consistencia de ambas y la homogeneidad. Recordemos para una mejor comprensión de esta problemática la vivencia de Husserl en cuanto a la dificultad de la percepción del Otro al confundir un maniquí como un visitante más del gabinete (Museo de cera de Berlín) a fines del siglo (XIX). Es decir, este ejemplo que describe Husserl del visitante que al entrar al museo de cera confunde a una muñeca que al saludarlo (programada para mover el brazo) con un ser humano real está relacionada con esta problemática de la posibilidad de experimentar al Otro. Este escenario más que solo ser una experiencia bochornosa fue la comprobación de un observador que estaba decidido a partir de la teoría de la mera evidencia y que por medio de ella cae en la tentación (en un primer plano) de la posibilidad de experimentar al Otro.

Recordemos que los griegos, definían al ser humano por la facultad y el placer de su visión elemento que entre otros contribuye a la posibilidad de ser visto. Sin embargo en su felicidad sin mundo entre los mundos, los dioses de Epicuro en alguna forma son un misterio por el hecho de no dejarse contemplar. En definitiva esto sucede por tratarse más bien de una antropología dualista, la que hace de la corporalidad el órgano desechable del sujeto.

La vista si bien es la constitución de la afección no tiene la consistencia ni menos aún una identidad garantizada como sustancia experimentada desde adentro. Recordemos que la visibilidad presupone la presencia del otro, es decir, la experiencia de que el otro me vea como yo lo veo. Que me identifique por y en mi apariencia, lo cual sería imposible si todos fuéramos iguales dotados de los mismos cuerpos. La única forma de que no nos identifiquen es poniéndonos una máscara y así parecernos a otro pasando desapercibido respecto a nuestra identidad. En definitiva el cuerpo es el único que por su parte hace del sujeto un 
objeto para otros sujetos. Desde la teoría del siendo sobre todo la circunstancia de que haya otros además de mí y que yo lo registre como posibilidad de objetivación de mi experiencia.

De allí que entonces parece ser que cada sujeto también es otro para aquellos otros cumpliendo el mismo servicio. Pero la verdad es que no parece obvio que con el descubrimiento metafísico de la corporalidad se descubra también la visibilidad. Respecto de la comprobación tardía de la visibilidad recordemos las palabras de Feuerbach: De que no somos solamente seres subjetivos, sino también objetivos; para las cosas, para los seres que están fuera de nosotros, que incluyen el álter ego del ser humano, somos lo que ellos para nosotros.

No sólo vemos también somos vistos ${ }^{2}$. (Ludwig Feuerbach: 301). De allí no se deduce de que se trata de una fenomenología pero al igual que el cartesianismo husserliano, contiene también el principio más esencial de un proceder fenomenológico: básicamente la anulación de un cartesianismo. Esa anulación más que reprochar el dualismo psicofísico reprocha más bien la fijación de la base de evidencia en el Cogito. Feuerbach expresa esa equivalencia de la certeza del cuerpo y certeza de si de esta manera: Si puedo poner en duda el cuerpo, me puedo poner en duda a mí mismo. No tengo más certeza de mí que de mí cuerpo; la inmediatez de mi cuerpo es también la de mí mismo. Sólo que cuando pienso en mí mismo no pienso inmediatamente en mi cuerpo, o por lo menos no en ciertas partes evidentes del mismo ${ }^{3}$. (Ludwig Feuerbach: 301). En esta frase llama la atención que se hable de mí certeza del cuerpo, es decir, de la experiencia interna del cuerpo pero de ninguna manera de que este cuerpo me trasmite la experiencia externa de otros. En definitiva que mejor experimento mental para comprender esta situación que el ser invisible para los otros. Sin embargo cabe preguntarse:

¿Qué acontecería si todo dependiera de nuestra conciencia y de nuestro recuerdo y nada de la perceptibilidad? Freud en sus investigaciones de lo profundo que pude tener una justificación terapéutica, resalto una formula al respecto del derecho a la propia oscuridad ${ }^{4}$. (Lou Andreas-Salomé, 1958: 115 y s.) El cuerpo de alguna manera impide que seamos invisibles. En la obra contemporánea más importante sobre la discusión de la corporalidad, Nachruf auf Ludwig Marcuse [Necrológica de Ludwig Marcuse], de Ludwig Marcuse, en la cual correlaciona la muerte y la renuncia a la auto exhibición afirmando que uno sólo vive mientras se arregla. Quien ya no toma en cuenta el ser mirado y ni siquiera este sutilísimo maquillaje, se deja caer en la muerte ${ }^{5}$. (Ludwig Marcuse, 1969: 221). La postura

2. Feuerbach, Ludwig, Nachgelassene Aphorismen, en Sämliche Werke, ed. De Wihelm Bolin y Friedrich Jodl, vol. X.

3. Feuerbach, Ludwig, Nachgelassene Aphorismen, en Sämliche Werke, ed. de Wilhelm Bolin y Friedrich Jodl. Vol. X.

4. Salomé, Lou Andreas, In del Schule bei Freud, Zúrich, 1958.

5. Marcuse, Ludwig, Nachruf auf Ludwig Marcuse, Munich, 1969. 
en cambio de Epicuro de que la muerte no importa ya que mientras vivimos la muerte no está y cuando aparece nosotros ya no estamos es simplemente una negación de lo que significa la corporalidad mortal. También se puede agregar que posiblemente Husserl hubiera renunciado al dilema del museo de cera respecto de la dificultad sobre la percepción del otro si hubiera privilegiado por ejemplo la experiencia de la muerte.

Esta frase nos sirve de introducción para entrar en nuestra próxima temática la experiencia de la muerte de otros como experiencia de la anulación de la apresentación. Con ello de alguna forma se estaría rebatiendo la afirmación de la posible experiencia de estar vuelto hacia la muerte como una originalidad interna de la conciencia. Esta argumentación estaría todavía dentro de la tradición cartesiana, en donde la idea de finitud la hallamos en nosotros antes de toda experiencia externa. Sin embargo el único modo de la idea de finitud es como negación de la idea de infinitud. Pero para Husserl esta idea anteriormente descrita es insostenible si la teoría de la constitución de la conciencia interna del tiempo, debería seguir siendo sostenible. Ya que en definitiva es una teoría de fabricación infinita del tiempo, que deja afuera el principio y el final como fenómenos, con ello demostrables fenomenológicamente.

Recordemos en este punto que una conciencia moral en Kant tiene que poner su principio más allá de la experiencia posible, tiene además la finalidad de recordar la importancia de la exterioridad esencial a determinados fenómenos, que se relacionan con la corporalidad del ser humano. Por otra parte, recordemos la primera Epístola de Juan en donde se expresa esa problemática más bellamente: En esto ha llegado el amor a su plenitud con nosotros: en que tengamos confianza en el día del Juicio, pues como él es, así somos nosotros en este cosmos. Se deduce de este párrafo de que los juzgados y los que juzgan forman parte de un cosmos o un mundo por ser sujetos físicos. Por lo tanto, la plenitud del amor solo es posible por formar parte de un mundo. Recordemos que los dioses de Epicuro no aman y es por no formar parte de ningún mundo.

Volviendo al tema del rasgo antropológico básico de la visibilidad está en su consistencia espacio tiempo es la que permite que aparezca la conciencia de sí. Si aceptamos lo que Husserl en la conferencia de Berlín afirma: Tomarse como humano es presuponer ya la validez del mundo, la presentación de hechos, imágenes y mitos, en el anuncio de lo humano parece resaltar sus miedos de que con el rotulo de la antropología se le pudiera quitar a la fenomenología el resultado del esfuerzo realizado con las reducciones. La verdad que en el pensamiento de Husserl es imposible describir y prescindir del mundo de la vida prescindiendo del ser humano cuyo mundo es. Esto sucede porque en el fondo Husserl tiende a un concepto formal del mundo de la vida. Lo común del mundo de la vida en Husserl hace años antes del estar-en-el- mundo de Heidegger es que: eliminan la dificultad del mundo objetivo como también su posibilidad de acceso teórico a él, dejándolo como secundario y dejándolo surgir solo en 
el desmontaje de una familiaridad originaria en el trato y que trascurre sin vacilaciones. La diferencia estriba que en Husserl este desmontaje del mundo de la vida es la ejecución de la perseverancia de la propia historia por hacer realidad la ciencia.

En cambio en Heidegger se presenta a causa de su originalidad como un estado de plenitud antes de que comience el empobrecimiento por objetivación, y más tarde la traslada al periodo anterior al ocultamiento del ser como metafísica. Una hipótesis distinta a la de Husserl y Heidegger que permite exponer la relación entre la fenomenología y la antropología puede ser la de introducir la pauta de que para la conciencia del cuerpo equiparándolo al hecho que se acerca a la invariancia de lo esencial. En otras palabras pensarse a sí mismo distinto de cómo uno es y averiguar las circunstancia en las cuales esto es posible pero sin dar por extraviada la identidad. Es decir, es el intento de pensar al Otro no como reduplicación del yo en otro cuerpo en otra posición espacio temporal, sino más bien por el hecho de encontrar una individualidad ajena basada en mi auto-representación.

Dentro de este marco tratemos de analizar la empatía con respecto a otro sujeto: ¿Solo es posible por poder pensarme como si yo fuera ese Otro? Esto se encuentra de alguna forma en los escritos de Husserl: Puedo pensar otros yo y pensarme distinto. Para mi ser-así sólo es vinculante el marco esencial general, que comprende todas las posibilidades de mi yo y de un yo en general. De un yo en general: porque toda posibilidad de otro yo tiene que mantenerse dentro del marco de la analogía, y hasta donde llega la analogía llega una unidad de la esencia. Toda posible variación que puedo imaginar en Otro, puedo imaginarla en mí mismo, y más originalmente ${ }^{6}$ (Edmund Husserl, 1922: 154-158).

Aquí también se deja en claro lo que se debe alcanzar: la posibilidad de imaginar al Otro en la identidad del marco esencial con el mío propio. Esta frase ya es un pilar fundamental de los escritos de la criptoteología fenomenológica, respecto de la certeza de sí y que dice que yo no soy y tengo la evidencia no sólo de que soy sino también de que soy necesario. Es impensable para mí que yo no sea. Por lo tanto, también es impensable que en general un yo no sea. También tengo la evidencia general: ningún yo puede imaginarse que un yo no sea. Soy el que soy. Husserl describe a la conciencia como un fluir, pero que no por ello se desintegra. Recordemos sus famosas metáforas de la corriente y del fluir de la conciencia que siempre están relacionadas por la intencionalidad de las configuraciones objetivas y de horizonte. Es decir, el yo ya no puede ser la sustancia que en algún momento le aseguro la identidad, pero tampoco se reduce por ello a la mera aglomeración de la nube de los datos del sensualismo. Siguiendo esta lógica de argumentación cabe recordar el viejo dilema no superado en la prueba ontológica de la existencia de Dios de Anselmo de Canterbury: el inferir del concepto la existencia. 
Respecto de este punto cabe recordar las palabras de Husserl: Allí hay que tener cuidado cuando se ha avanzado tanto como lo hemos hecho nosotros. Naturalmente, no es que la empatía sea y pueda ser precedida por fantaseos sobre mi yo mediante los cuales yo pudiera adquirir otros yoes concretos. Fantasear sobre algún fragmento de mi vida ¿eso ya crea, en lugar del que soy yo, el yo como si fuera otro yo? Tendría que pensar hasta el final consecuentemente el fragmento de ficción. Pero si tengo Otros fácticamente y por consiguiente como posibilidades, puedo fantasear sobre mí, etcétera ${ }^{7}$ (Edmund Husserl, Husserliana XIV: 160). El dilema estriba según Husserl de que otro, pero no para mí y no como magnitud de referencia de un mundo en común, el que yo sin embargo podría seguir teniendo como idea de posible objetividad. Este es el trama mental que nos ha dejado Husserl en su experimento mental de que pudieran llegar a existir muchos sujetos puros para sí mismos, pero en una vida sin mundo ${ }^{8}$. (Edmund Husserl, Husserliana XV: 32).

En la obra relativo a las Conferencias de París sobre la dependencia del ser de todo ente, primero de todos los sujetos trascendentales con respecto a mí y luego de mí mismo con respecto a ellos, anoto primero esta reflexión: Esto hay que pensarlo seriamente, no parece haber una respuesta posible? . (Edmund Husserl, Husserliana XV: 32). Por medio de este ejemplo se nos complicó el panorama y volvemos a la pregunta: ¿Cómo sé que aspecto tengo?, si mi experiencia de mi cuerpo es también una experiencia del otro. Puedo convertir (por principio kinestésicamente) todos los lados posteriores del mundo y de la Luna, en mis lados anteriores, excluyendo mi propia parte posterior. Esto simplemente refleja la inaccesibilidad parcial del cuerpo propio para la experiencia de sí, de los ángulos muertos desde la óptica y de las percepciones táctiles. Recordemos que incluso nosotros vivimos en medio de efectos visuales, espejos, y fotografías gracias a los medios de comunicación los que a su vez nos hacen olvidar el hecho de las complicaciones de la percepción de sí. En realidad la visibilidad es diferente si no se le da alcance mediante la tactilidad. ¿O la tactilidad va más lejos que la visibilidad o es más confiable? Husserl integra este tocar y ver en una percepción total en donde el cuerpo es un órgano total para sí mismo. ${ }^{10}$

Lo importante de esta sensación de sí mismo es la imposibilidad de separarse del cuerpo propio o desactivarlo por algún motivo y a la vez de mantenerlo consigo en cualquier auto traslado imaginario hacia un allí en relación con un aquí. Husserl afirma que no puedo empujarme, esencialmente, como si mi cuerpo pudiera seguir siendo sin mí y yo seguir siendo el que empuja sin cuerpo ${ }^{11}$ (Edmund Husserl: 299 y s.). En realidad la diferencia entre la percepción táctil y óptica

7. Husserl, Edmund, Husserliana XIV.

8. Husserl, Edmund, Husserliana XV.

9. Husserl, Edmund, Husserliana XV

10. Husserl, Edmund, "Universale Geisterwssenschaft als Anthropologíe. Sinn einer Anthropologíe", noviembre-diciembre de 1932, Husserliana XV.

11. Husserl, Edmund, Husseliana XV. 
describe la restricción de la libertad imaginativa respecto del cuerpo: Pero podría imaginarme también que visualmente mi cuerpo se va volando, mientras las manifestaciones transcurren de tal modo que yo sigo viendo desde aquí y el cuerpo acaba desapareciendo en el horizonte con las modificaciones y las reducciones que ya conocemos de la perspectiva. Visualmente es una representación posible, pero el cuerpo no tiene sólo una constitución visual y plantea exigencias a la manifestación táctil ${ }^{12}$ (Edmund Husserl, 1918: 418). En definitiva el cuerpo es afectado por su conjunto se debe a la unidad que lo constituye como órgano total para sí mismo, por lo cual se experimenta a sí mismo en la correlación pasiva con cada uno de sus formas de comportamientos activos.

Para profundizar en esta temática recordemos al fundador de la fisiología de los sentidos, Johannes Müller, que observo el fenómeno de la sensación homogénea. En el esbozo de su física de los nervios expone este fenómeno a partir de un sustrato nervioso unitario de todos los órganos de los sentidos. Lo importante que para Müller, el espacio objetivo no es más que la proyección al mundo exterior de esta autoconcepción homogénea, como también de la subordinación a ella de todas las sensaciones especiales. Por ejemplo, el dolor presupone la sensación total homogénea del cuerpo. Pero como localización fantasma, la localización y la proyección están orientadas en un espacio próximo del ya no de la corporalidad propia y todavía no de su exterioridad. Sin embargo, en este punto recordemos el experimento del miembro fantasma que después de haber sido amputado a un paciente, este igual sentía un dolor localizado en un miembro inexistente. Recordemos que Platón va a considerar la vida contemplativa como una vida esencialmente privilegiada, en detrimento del bios políticos.

Es por ello, que la esfera de los asuntos humanos quedara relegada a un segundo plano. Esta retirada de la mundanidad, de la vida cotidiana era un rasgo esencial de la contemplación. Lo que importaba era la skchole o libertad de actividad política. Un ejemplo de ello era lo que preconizaba Epicuro (el abandono del compromiso político activo) para mantener una existencia de retiro contemplativo, en la que el filósofo se mantiene física y psíquicamente apartado del tumulto de la vida pública. Lo contrario de ello en cambio, sería la vida activa que pasaría a caracterizarse, negativamente, por la askolia u in-quietud. Al cambiar la preferencia del modelo cambia también la consideración sobre los sujetos. Por otro lado para Platón la propia etimología de la palabra teoría está ligada a la contemplación de lo divino. El termino deriva de theoros, que era el nombre que se daba al enviado especial que la polis mandaba o también como espectador de los festivales sagrados, o bien para consultar el oráculo de Delfos. El Theros era el intermediario entre la polis y las palabras de los dioses, de las cuales tenía que dar detalladas cuentas. Este intermediario de dedicaba a mirar, contemplar, sin intervenir en el acontecimiento transformándose mediante la

12. Husserl, Edmund, Husserliana XIII, [texto de 1918]. 
contemplación en una criatura próxima a los dioses. Platón incluye un cambio esencial- que sería recogido posteriormente por Bentham en la estructura del Panóptico- y es la primacía de la vista por sobre la acción.

Sin embargo resulta absurdo afirmar de la tradición filosófica que el observador del mundo solitario y libre de escrúpulos existenciales jamás hubiera caído en la reflexión. Recordemos el esclavo virgen de la teoría que Platón presentada en el Menón, al que se lo puede llevar fácilmente a comprender la geometría, es decir la anamesis platónica puede describir como el esclavo puede introducir la ley pura en la figura empírica. Sin embargo, no habría mayéutica capaz de llevarlo a recordar el acto y el proceso de esa visión preexistente de las ideas como tal. Gracias al recuerdo se puede llevar a alguien a la geometría pero ni la elocuencia de Sócrates podría empujar a la teoría de las ideas y a su fundamentación.

Pareciera que la visión prenatal de las ideas del alma platónica debe pensarse tan lejos de toda reflexión como la capacidad del esclavo despierto para las matemáticas. Pero, quien tiene las ideas ante sí, sería incapaz de autoconocimiento y a su vez no lo necesitaría. En este punto el platonismo hace difícil sostener al Sócrates del autoconocimiento. En la Biblia de alguna manera se elogia aquellos que no ven y obstante creen, porque en el fondo es por el testimonio de los que han visto.

En cambio, lo que se denomina conocimiento del mundo y empatía, se basa en el refinamiento de la capacidad de tener conciencia de que se es perceptible por los otros para los otros. Sin embargo lo que vemos es a su vez interpretado dependiendo de la cultura ósea del contexto. Por ejemplo, las formas de la desnudez no toleradas difieren en las distintas culturas, pero las formas de vestirse también y pueden pasar fácilmente de lo tolerable al ridículo. La vestimenta tiene una capacidad de manifestar conformidad e incluso su reprobación puede incluso penalizarse dependiendo el país en cuestión. Stendhal relata en sus Recuerdos de egotismo, publicado por primera vez en 1892, que conoce en el salón de madame Cabanis a una joven, de la que su estudioso Henri Martineau supone que se trata de madame Victor de Trasy. Él nunca fue su amante pero supone que la dama se hubiera alegrado si hubiera tenido la inteligencia de haberle dicho que la amaba.

Toda la problemática se reduce a la siguiente frase: que no la amo lo suficiente para poder olvidar lo feo que soy, aunque ella lo había olvidado. La situación del joven literato y la dama es simétrica, pero solo la seguridad de que ella ya no se percataba de su fealdad podría haber hecho que la olvidara. El joven sabía que ella se había olvidado de su aspecto, y eso quería decir sin duda que estimaba su persona. Pero el joven fue incapaz de creerlo. El sujeto cuya percepción lo era todo no le basto la irrelevancia de su aspecto. El necesitaba un testigo de que su exterior no era indicio de su verdad externa, pero por otro lado no pudo avalar la 
calidad de ese testigo. Eso es justamente lo que siente por la insuficiencia de su amor. Lo que en el fondo resalta este ejemplo es la constitución del otro sujeto en cuanto están en juego las necesidades más profundas de la autoconstitución. En el fondo es la misma inseguridad que percibe el protagonista Tom Cruise en la película abre tus ojos con la gran actriz Penélope Cruz.

De alguna forma pareciera que la empatía es la capacidad que estabiliza la confianza de que el otro me vea solamente como si yo mismo me hubiera instalado en su lugar para mirarme, que tenga que tomar lo exterior por lo interior, el síntoma por lo profundo. Otro ejemplo de la dificultad que encierra la reflexividad de la constitución de sí mismo es el mito de Narciso tal como lo concibe la figura en la psicología: el ver su reflejo en la fuente se vio a sí mismo y en su delirio erótico se precipito hacia el reflejo y la muerte. Pero el mito seguramente no quiso decir tal cosa, ya que Narciso en realidad no reconoce el reflejo como el suyo. Más bien el erotismo, lo había seducido y arrastrado a la perdición en el desconocimiento de la belleza ajena que tenía a su alcance. Esto lo encontramos también reflejado en la famosa novela de Oscar Wilde, El Retrato de Dorian Gray, en la cual el protagonista, como hombre muy bien parecido, admira su retrato recién pintado y expreso el deseo de que sea el lienzo que en su lugar envejezca. Un diablo lo escuchó y le garantizó su deseo. Es así como vende su alma, para no envejecer. Con angustia, Dorian, se vuelve testigo de los estragos que, al retrato, infligen su vida disipada y alma despiadada y narcisista. Pero mientras que él no envejece o cambia, el retrato luce como monstruosidad repugnante. En la trama de la novela, Wilde condensa, en la metáfora provista por el retrato, la interacción de motivos narcisistas y grandiosos. Plasmados entre sí en la necesidad de la belleza eterna y en la abolición del proceso del envejecimiento natural. Oviedo afirma de que no es el narcisismo del amor propio original, sino el primer descubrimiento de una satisfacción que termina siendo un engaño por desconocer que el sí mismo es uno sólo. ${ }^{13}$ (Ovidio, Metamorfosis, III, 346-348: 407-510). En ese texto sale que Narciso no se reconoce: se queda mirándose inmóvil como si fuera una imagen de mármol, es decir que omite identificarse porque la imagen carece de movimiento. Admira esas cualidades pero no se admira a sí mismo como portador de esas cualidades ${ }^{14}$ (Ibíd.: 424). El poeta se traslada de la tercera persona a la segunda como advirtiéndole a Narciso de que el reflejo depende de él mismo. ${ }^{15}$ (Ibíd.: 432-436). Narciso no cree en el fondo que entre la apariencia y la realidad este todo ${ }^{16}$. (P. Hadot, 1976: pp. 81-108). Pero también se puede observar la problemática de definición si tomamos el existencialismo e insertamos el genitivo de la sociedad para obtener una desposesión del individuo con respecto a sí mismo. Inautenticidad es sólo uno de los términos para indicar

\footnotetext{
13. Ovidio, Metamorfosis, III, 346-348: 407-510).

14. Ovidio, Metamorfosis, III, p. 424.

15. Ovidio, Metamorfosis, III, p. 432-436

16. Hadot P., "Le Mythe de Narcisse et son Interprétation par Plotin", en Nouvelle Revue de la Psychanalyse, núm. 13, 1976.
} 
que la vida puede actuar, y es bastante irrelevante si soy yo el que la actúa, o es otro o son los Otros.

Esta exterioridad propia puede funcionar también de una manera reflexiva por el hecho de que haya otros sistemas sensoriales y portadores de intenciones que podrían afectarme. Max Scheler ha deducido que esta posible posición externa del sujeto en relación a sí mismo, la objetivación de su cuerpo y de sus movimientos, de la contingencia de su posición en el espacio, en el universo, es más que una posición externa. Afirmando que es la posición del individuo con respecto a sí misma como una posición trascendente respecto a sí misma. Y al que el ser humano puede elevarse por encima de sí mismo como ser vivo, partiendo de un centro situado, por decirlo así, allende el mundo tiempoespacial, convertir todas las cosas, y ente ellas también a sí mismo, en objeto de su conocimiento ${ }^{17}$. (Max Scheler: 1976: pp. 64 y s.). Por ello el ser humano es en definitiva el ser superior a sí mismo como ser vivo y al mundo. Para ello debe ejecutar actos desde una situación que hace del mundo en su plenitud espacial y temporal su objeto, una posición que no pertenece ella misma a este mundo y que no es determinable en él espacial y temporalmente. Esta posición sólo puede residir en el fundamento supremo del ser mismo ${ }^{18}$ (ibíd.: p. 65).

De alguna forma se trata de una autoobjetivacion, carente de todo fundamento. Se basa en que su origen está en que el fenómeno de la exterioridad propia, de la reflexibilidad en general, no se considera desde una visión genética. Una visión más bien eidética impide admitir el aspecto genético. De alguna forma este es el sitial en donde el ser humano encuentra su diferencia insuperable en relación a los demás seres vivos.

Cabe mencionar que entre las diversas definiciones que Nietzsche le entregó al hombre antes del comienzo del superhombre está también la de que es un "animal opaco"19 (Friedrich Nietzsche, 1949) y no se refiere a él de una manera peyorativa. En el Preludio de una filosofía del futuro describe con un gran respeto las maneras de disfraz del ser humano con las que éste se sustrae al zarpazo de la curiosidad. Este autor agrega que impermeabilizar la superficie, sustraerse a la mirada que los otros lanzan a las profundidades del dolor, es condición de la alegría. Nietzsche recuerda el cinismo de Hamlet y al abate Galiano, que le demuestran que es de una humanidad refinada tener respeto ante la máscara y no andar practicando la psicología y la curiosidad en el lugar equivocado. La cara es también el lugar de la vulnerabilidad, la parte del cuerpo no protegida de la opacidad, el órgano que delata la decadencia, edad, imbecibilidad, el asco.

17. Scheler, Max, Die Stellung des Menschen im Kosmos, 1928, en Gesammelte Werke, vol. IX, pp.38 y s. [trad. Esp.: El puesto del hombre en el cosmos, $12^{\circ}$ ed. Trad. De José Gaos, Buenos Aires, Losada, 1976.

18. Scheler, Max, (trad. Esp.: El puesto del hombre en el cosmos, $12^{\circ}$ ed. Trad. De José Gaos, Buenos Aires, Losada, 1976, p. 65).

19. Nietzsche, Friedrich, Jenseits von Gut und Böse, IX, \& 291 (Musarion-Ausgabe, vol. XV, p. 255)

[trad. Esp: Más allá del bien y del mal, Buenos Aires, Aguilar, 1949]). 
Pero volvamos a la importancia de la mirada pero a su vez de su problemática. Husserl afirma que en la experiencia de la intersubjetividad se necesitó que el otro tenga allí los objetos de mi mundo (los mismos que yo tengo) aunque en otro matiz, se base en la primacía de la corporalidad propia. Sin embargo, esto queda descartado para la experiencia directa de la mirada ya que la experiencia de la mirada no es una cuestión de poder comparar percepciones. Es decir, puedo imaginarme que para generar la percepción del otro podría dirigirme al lugar donde lo percibo para percibir todo desde allí pero es imposible que pueda ponerme de tal manera en el lugar del otro (y que pudiera verme a los ojos).

Este límite del fantaseo Husserl lo resume así: Que al mismo tiempo bien puedo imaginarme una transformación, una transformación física, de mi propio cuerpo, que mi propio cuerpo estuviera ahora constituido exactamente como el del otro. Claro que entonces tomo mi cuerpo y el suyo como meros cuerpos de la naturaleza ${ }^{20}$ (Edmund Husserl, 1931: 251). Esto es válido también para la percepción ajena, ya que me puedo imaginar, con ayuda de un espejo y la imagen, lo que el otro ve cuando me ve, pero es imposible llegar a la última profundización de la ficción e imaginarme la mirada del otro en mi ojo. Si la experiencia del otro es problemática, la experiencia de sí lo es todavía más. En caso de ser esta la base de la experiencia del otro, solo lo sería en la comprensión trascendental del yo. De alguna forma se lo que es un yo en general mejor que lo que es mi propio yo. Wittgenstein lo interpreta diciendo que puedo saber lo que el Otro piensa pero no lo que yo pienso ${ }^{21}$ (Ludwig Wittgenstein, 1988: 506 y 507). Este sería el triunfo de la premisa general de la filosofía del lenguaje, en la cual el lenguaje está detrás de todo lo que puede tomar estado de conciencia.

Así se llega a la formulación de que el ser humano no sabe lo que es, lo que piensa, ni sabe lo que sabe. De allí nace la máxima de que el ser humano se define por hacer algo sólo para saber si puede hacerlo. Es la continuación antropológica del postulado de comienzos de la modernidad según la cual lo verdadero y lo hecho son intercambiables (verum et factum convertuntur). Así se convierte el ser humano en opacidad para sí mismo en donde su presentización se encuentra más bien como resultado de su historia. Nietzsche lo formula diciendo que no sólo el pensamiento propio nos es copresente, sino que ese pensamiento es el síntoma superficial de un proceso intrasubjetivo cuyas eurupciones no solo percibimos sino que además somos. Pero ¡Qué sabe el individuo en realidad de sí mismo!

¿Sería éste capaz de percibirse una vez completo, tendido como en una vitrina iluminada? ¿Y acaso la naturaleza no le oculta la mayor parte, incluso sobre su

20. Husserl,Edmund, Husserliana XV, p. 251, nota (texto sustituido por corrección), "Zur Lehre von der Einfühlung", Agosto de 1931.

21. Wittgenstein, Ludwig, Philosophie Untersuchungen. Schriften I, Francfort, 1960, p. 534 [trad. Esp.: Investigaciones filosóficas, trad. De Alfonso Garcia Suárez y Ulises Moulines, Barcelona, Critica, 1988. 
propio cuerpo, para encerrarlo en una orgullosa conciencia ilusoria, lejos de las sinuosidades de los intestinos, el rápido fluir de los torrentes de sangre, las intrincadas trepidaciones de las fibras? ${ }^{22}$ (Friedrich Nietzsche, 1873: 77). Es decir, la naturaleza nos cero la puerta, y la fatalidad parece comenzar amenazando la curiosidad que fuera capaz una vez de mirar hacia afuera y hacia abajo por una hendija el recinto de la conciencia. A partir de todo ello se puede concluir que toda antropología, también la que lo niega, es en esencia histórica. En definitiva no es una desgracia trascendental que la experiencia de sí sea una experiencia indirecta histórica y cultural.

Husserl habla de un mundo circundante común partiendo de objetos espirituales (término de la época), es decir de los objetos culturales, que aparecen a su vez como universales. Universales, es decir intersubjetivos y como dependientes de las ciencias del espíritu. Incluso agrega que muchos de mis convicciones pueden provenir de la experiencia de los otros y también puede ser que reconozcan que algunas de sus experiencia provengan de la mía. Y que haga que el individuo dependa del resultado de los otros sobre él. De alguna forma la falta de obtener claridad sobre sí mismo sin hacerlo condicionado por los deseo a de objetivación de los demás sobre él. Es así que por falta de claridad de sí, el ser humano no es capaz de apreciar a los otros como instancia ni de rechazarlos como tal.

Esa es la hipótesis de Nietzsche al decir que lo que sabemos y recordamos sobre nosotros mismos no es tan primordial como se afirma para la felicidad de nuestras vidas ya que: Pero llega el día en que lo que los demás saben de nosotros o se figuran saber se apodera de nuestro ánimo, y entonces reconocemos que es lo que más importa. Anunciando que es preferible una conciencia intranquila que una mala reputación.23 (Friedrich Nietzsche, 2001: 54). En síntesis, el mundo a partir de la incertidumbre de la garantía de la realidad del Medioevo, tuvo que fortalecerse mediante el sujeto cartesiano. Es así como la relación entre subjetividad-objetividad queda invertida con Descartes. A partir de todos los descubrimientos técnicos, tecnológicos estamos más seguros del mundo que de un yo que ha resultado débil e infiltrado.

En definitiva la crisis más que una crisis de la modernidad por predisponer de bases inseguras pareciera ser más bien una ilusión. Es decir, la crisis no es la del acceso del sujeto a los objetos sino más bien a la inaccesibilidad del sujeto para sí mismo. Pero siguiendo a Friedrich Nietzsche a causa de la percepción en su opacidad, siéndolo en consecuencia también para los otros. En definitiva esta idea se la puede vincular con su descubrimiento de que el autoconocimiento no existe lo cual deja a la conciencia a la merced del veredicto de los otros aunque no hay razón de suponer que aquellos están más facultados para ese conocimiento. En verdad solo tienen el poder de sugerir e imponer algo que

22. Wittgenstein, Friedrich, "Uber Wahrheit und Lüge im aussermoralischen Sinne” (1873), MusarionAusgabe, vol. VI. 
no se puede refutar por depender entre otros factores de un contexto cultural determinado. Por otro lado cabe recalcar la dificultad actual de la psicología en poder definir al yo y esto sucede entre otros fenómenos contextuales y culturales también por la irrupción del inconsciente. Edmund Husserl en definitiva fue un visionario al sospechar de la intersubjetividad, del yo como también en parte de la psicología.

\section{Bibliografía}

Feuerbach, Ludwig. Nachgelassene Aphorismen, Leipzig: en Sämliche Werke, Ed. De Wihelm Bolin y Friedrich Jodl, vol. X. 1849.

Salomé, L. In del Schule bei Freud, Zúrich. 1958.

Marcuse, L. Nachruf auf Ludwig Marcuse, Munich. 1969.

Hadot P. Le Mythe de Narcisse et son Interprétation par Plotin, en Nouvelle Revue de la Psychanalyse, núm. 13. 1976.

Husserl, Edmund. Die Notwendigkeit der Ichexistenz, (Husserliana XIV). 7 y 8 de Enero 1922.

Husserl, Edmund., Universale Geisterwssenschaft als Anthropologíe. Sinn einer Anthropologíe, Husserliana XV. Noviembre- Diciembre 1932.

Husserl, Edmund. Zur Lehre von der Einfühlung. Husserliana XV. Agosto 1931.

Nietzsche, Friedrich. La gaya ciencia, Buenos Aires, El Ateneo. 2001.

Nietzsche, Friedrich. Más allá del bien y del mal, Buenos Aires, Aguilar. 1949.

Scheler, Max. El puesto del hombre en el cosmos, Buenos Aires, Losada. 1976.

Wittgenstein, Ludwig. Philosophie Untersuchungen. Schriften I, Francfort. 1960.

Wittgenstein, Ludwig. Uber Wahrheit und Lüge im aussermoralischen Sinne, MusarionAusgabe, vol. VI. 1873. 